Y. Kakuda

Nagoya Math. J.

Vol. 48 (1972), 159-168

\title{
SATURATED IDEALS IN BOOLEAN EXTENSIONS
}

\author{
YUZURU KAKUDA
}

0. Introduction. Let $\kappa$ be an uncountable cardinal, and let $\lambda$ be a regular cardinal less than $\kappa$. Let $I$ be a $\lambda$-saturated non-trivial ideal on $\kappa$. Prikry, in his thesis, showed that, in certain Boolean extensions, $\kappa$ has a $\lambda$-saturated non-trivial ideal on $\kappa$. More precisely,

THEOREM (Prikry [8]). Let $\kappa, \lambda$ and $I$ be as above. Let $\mathscr{B}$ be a $\lambda$ saturated complete Bollean algebra. Let $J \in V^{(\mathfrak{B})}$ such that, with probability $\mathbf{1}, J$ is the ideal on $\check{\kappa}$ generated by $\check{I}$. Then, it is $\mathscr{B}$-valid that $J$ is a $\breve{\lambda}$ saturated non-trivial ideal on $\check{\kappa}$.

The following questions naturally arise; 1) If $I$ is $\kappa$-saturated $\left(\kappa^{+}-\right.$ saturated), does $J$ remain $\kappa$-saturated $\left(\kappa^{+}\right.$-saturated)? 2) If $\operatorname{sat}(\mathscr{B})=\kappa$, what is the saturatedness of $J$ ?

For 1), we obtain the following theorem.

THEOREM 1. Let $\kappa$ and $\lambda$ be as above. Let $\gamma$ be a regular cardinal such that $\lambda \leq \gamma \leq \kappa^{+}$, and let $I$ be a $\gamma$-saturated non-trivial ideal on $\kappa$. Let $\mathscr{B}$ be a $\lambda$-saturated complete Boolean algebra. Then, it is $\mathscr{B}$-valid that $J$ is $\gamma$-saturated.

For 2), we get the following theorems.

THEOREM 2. Let $\kappa$ be an uncountable cardinal, and $I$ be a $\kappa$-saturated non-trivial ideal on $\kappa$. Let $\mathscr{B}$ be a homogeneous complete Boolean algebra such that $\operatorname{sat}(\mathscr{B})=\kappa$. Then, it is $\mathscr{B}$-valid that $J$ is not $\kappa$-saturated.

THEOREM 3. Let $\kappa$ be a measurable cardinal, and $I$ be a non-trivial prime ideal on $\kappa$. Let $\mathscr{B}$ be a homogeneous complete Boolean algebra such tnat $\operatorname{sat}(\mathscr{B})=\kappa$. Then, it is $\mathscr{B}$-valid that $J$ is not $\kappa^{+}$-saturated.

We will prove the above theorems as applications of a certain useful lemma, which will be proved in $\S 4$.

Received February 7, 1972. 
We assume that the reader is familiar with the Scott-Solovay Booleanvalued models for set theory.

\section{Saturated ideals.}

1.1. Let $\lambda$ be a cardinal. Let $\mathscr{B}$ be a Boolean algebra. We say that $\mathscr{B}$ is $\lambda$-saturated if, for any pairwise disjoint family $\left\{b_{\alpha}\right\}_{\alpha<\lambda}$ of $\mathscr{B}$, there exists some $\alpha<\lambda$ such that $b_{\alpha}=0$. Clearly, if $\lambda<\gamma$ and $\mathscr{B}$ is $\lambda$ saturated, then $\mathscr{B}$ is $\gamma$-saturated. $\operatorname{sat}(\mathscr{B})$ denotes the least cardinal $\lambda$ such that $\mathscr{B}$ is $\lambda$-saturated.

The following lemma is well-known.

LEMma. If $\operatorname{sat}(\mathscr{B}) \geq \aleph_{0}$ then sat $(\mathscr{B})$ is an uncountable regular cardinal.

1.2. Let $\kappa$ be an uncountable cardinal. Let $I$ be an ideal on $\kappa$. $I$ is called non-trivial if ;

1) $I$ is non-principal, that is, $\{\alpha\} \in I$ for all $\alpha<\kappa$.

2) $I$ is $\kappa$-complete, that is, if whenever $\eta<\kappa$, and $\left\{A_{\alpha}, \alpha<\eta\right\}$ is a family such that $A_{\alpha} \in I$ for each $\alpha<\eta$, then $\bigcup_{\alpha<\eta} A_{\alpha} \in I$.

Let $I$ be an non-trivial ideal on $\kappa$. We can form the quotient algebra $\mathscr{A}=P(\kappa) / I$. If $\mathscr{A}$ is $\lambda$-saturated, we say that $I$ is $\lambda$-saturated.

Solovay proved the following theorem.

THEOREM (Solovay [5]). Suppose that $\kappa$ has $\kappa$-saturated non-trivial ideal on $\kappa$. Then, $\kappa$ is the $\kappa$-th weakly inaccessible.

For more informations about saturated ideals, the reader may refer to Kunen [1], Kunen-Paris [2] and Solovay [5].

\section{The ultrapowers inside $V^{(\mathscr{)})}$.}

In this section, we restate the necessary results from Solovay [5].

From 2.1 to 2.3, we fix a transitive model $M$ of $Z F C$, and an ordinal $\rho$ in $M$.

2.1. Let $\mathscr{U}$ be a subset of $P(\rho) \cap M$. We say that $\mathscr{U}$ is an $M$ ultrafilter on $\rho$ if :

(1) $\mathscr{U}$ contains no singletons.

(2) If $A \in \mathscr{U}, B \in P(\rho) \cap M$, and $A \subseteq B$, then $B \in \mathscr{U}$.

(3) If $A \in P(\rho) \cap M$, then either $A \in \mathscr{U}$ or $\rho-A \in \mathscr{U}$. 
(4) Let $\eta<\rho$. Let $\left\langle A_{\xi}, \xi<\eta\right\rangle$ be a sequence such that $A_{\xi} \in \mathscr{U}$ for each $\xi\left\langle\eta\right.$ and $\left\langle A_{\xi}: \xi\langle\eta\rangle \in M\right.$. Then, $\bigcap_{\xi<\eta} A_{\xi} \in \mathscr{U}$.

The concept of $M$-ultrafilter is due to Kunen [1]. The reader should note that this definition somewhat differs from that of Kunen.

2.2. Let $\mathscr{U}$ be an $M$-ultrafilter on $\rho$. We define an equivalence relation $\simeq$ on $M \cap M^{\rho}$ as follows; for $f, g \in M \cap M^{\rho}$ let

$$
f \simeq g \quad \text { iff }\{\alpha<\rho ; f(\alpha)=g(\alpha)\} \in \mathscr{U} .
$$

We denote by $[f]$ the Scott equivalence class of $f$ with respect to $\simeq$.

Next, we put $N=\left\{[f] ; f \in M \cap M^{\rho}\right\}$. We define a binary relation $E$ on $N$ as follows; Let $f, g \in M \cap M^{\rho}$.

$$
[f] E[g] \quad \text { iff }\{\alpha<\rho ; f(\alpha) \in g(\alpha)\} \in \mathscr{U} \text {. }
$$

It is clear that the definition of $E$ does not depend on the choice of $f$ and $g$. The relational structure $\langle N, E\rangle$ is denoted by $\operatorname{Ult}(M, \mathscr{U})$.

2.3. Lemma 1 (Los). Let $\phi\left(v_{0}, \cdots, v_{n_{-1}}\right)$ be a set-theoretical formula, and let $f_{0}, \cdots, f_{n-1}$ be elements of $M \cap M^{\rho}$. Then,

$$
N \vDash \phi\left(\left[f_{0}\right], \cdots,\left[f_{n-1}\right]\right) \quad \text { iff }\left\{\alpha<\rho ; M \vDash \phi\left(f_{0}(\alpha), \cdots, f_{n-1}(\alpha)\right)\right\} \in \mathscr{U} .
$$

Let $x$ be in $M$. We define $c_{x} \in M \cap M^{\rho}$ by $c_{x}(\alpha)=x$ for all $\alpha<\rho$, and define $c: M \rightarrow N$ by $c(x)=\left[c_{x}\right]$.

LEMMA 2. $c$ is an elementary embedding.

In the remainder of this section, $\kappa$ will be uncountable cardinal, and $I$ a $\kappa^{+}$-saturated non-trivial ideal on $\kappa$.

2.4. We form the quotient algebra $\mathscr{A}=P(\kappa) / I$. Let $A \in P(\kappa)$. We denote by $[A]$ the element of $\mathscr{A}$ represented by $A$.

LEMMA $3 .^{1)} \mathscr{A}$ is complete.

Let $V^{(\infty)}$ be the Scott-Solovay $\mathscr{A}$-valued model. We assume that $V^{(\infty)}$ is separated.

2.5. We define an element $\mathscr{U}$ of $V^{(\mathscr{A})}$ as follows;

$$
\|\check{A} \in \mathscr{U}\|=[A] \quad \text { for each } A \in P(\kappa) \text {. }
$$

1) See Sikorski, Boolean algebras, Springer-Verlag, Berlin, 1960 p.65, 21.3. 
LEMMA 4. With probability 1. $\mathscr{U}$ is a $\check{V}$-ultrafilter on $\check{\kappa}$.

By Lemma 4, we can form Ult $(\check{V}, \mathscr{U})$ inside $V^{(\infty)}$.

LEMMA 5. Let $f_{0}, \cdots, f_{n-1} \in V^{x}$. Let $\phi\left(v_{0}, \cdots, v_{n-1}\right)$ be a set-theoretical formula. Then,

$$
\left\|\operatorname{Ult}(\check{V}, \mathscr{U}) \vDash \phi\left(\left[\check{f}_{0}\right], \cdots,\left[\check{f}_{n-1}\right]\right)\right\|=\left[\left\{\alpha<\kappa ; \phi\left(f_{0}(\alpha), \cdots, f_{n-1}(\alpha)\right] .\right.\right.
$$

The lemma is easily proved by using Lemma 1 and the following sublemma.

SUBLEMMA. Let $x_{0}, \cdots, x_{n-1} \in V . \quad$ Let $\phi\left(v_{0}, \cdots, v_{n-1}\right)$ be a set-theoretical formula. Then,

$$
\left\|\check{V} \vDash \phi\left(\check{x}_{0}, \cdots, \check{x}_{n-1}\right)\right\|=\mathbf{1} \quad \text { iff } \phi\left(x_{0}, \cdots, x_{n-1}\right) .
$$

LEMMA 6. Let $x \in V^{(\mathscr{x})}$. Suppose that $\|x \in \operatorname{Ult}(\check{V}, \mathscr{U})\|=1$. Then, for some $f \in V^{\kappa},\|x=[\check{f}]\|=\mathbf{1}$.

LEMMA 7. With probability 1, Ult $(\check{V}, \mathscr{U})$ is well-founded.

2.6. By Lemma 7, there exists a transitive class of $V^{(*)}, N$, and an isomorphism $\psi: \operatorname{Ult}(\check{V}, \mathscr{U}) \rightarrow N$ inside $V^{(\infty)}$. Let $f \in V^{(x)}$. Let $\psi(f)$ be the element of $V^{(\mathscr{x})}$ such that $\|\psi(f)=\psi([\check{f}])\|=1$. We put $x^{*}=\psi\left(c_{x}\right)$.

LEMMA 8. (1) With probability $\mathbf{1}, N$ is a transitive class containing all ordinals.

(2) Let $f_{0}, \cdots, f_{n-1} \in V^{k}$. Let $\phi\left(v_{0}, \cdots, v_{n-1}\right)$ be a set-theoretical formula. Then,

$$
\left\|N \vDash \phi\left(\psi\left(f_{0}\right), \cdots, \psi\left(f_{n-1}\right)\right)\right\|=\left[\left\{\alpha<\kappa ; \phi\left(f_{0}(\alpha), \cdots, f_{n-1}(\alpha)\right)\right] .\right.
$$

(3) Let $\|x \in N\|=1$. Then, $x=\psi(f)$ for some $f \in V^{x}$.

(4) If $\alpha<\kappa, \alpha^{*}=\check{\alpha}$.

(5) $\left\|\kappa^{*}>\check{\kappa}\right\|=\mathbf{1}$.

LEMma 9. With probability $\mathbf{1}, N$ contains all $\check{\kappa}$-sequences of $N$ in $V^{(\mathscr{A})}$.

Proof. Let $s \in V^{(\alpha)}$ be such that $\|s ; \breve{k} \rightarrow N\|=1$. For each $\alpha<\kappa$, we can choose $f_{\alpha} \in V^{x}$ such that $\left\|s(\check{\alpha})=\psi\left(f_{\alpha}\right)\right\|=1$. Let $\psi(g)=\kappa$. We define $f \in V^{*}$ by $f(\alpha)=\left\langle f_{\beta}(\alpha): \beta\langle g(\alpha)\rangle\right.$.

Clearly, $\| N \vDash \psi(f)$ is a $\check{\kappa}$-sequence $\|=\mathbf{1}$. We claim that $\|\psi(f)=s\|=$ 1. Now, choose $h_{\alpha} \in V^{\kappa}$ so that $\left\|(\psi(f))(\check{\alpha})=\psi\left(h_{\alpha}\right)\right\|=\mathbf{1}$ for each $\alpha<\kappa$. 
Then, $\| N \vDash \psi\left(h_{\alpha}\right)$ is the value of $\check{\alpha}$ by $\psi(f) \|=\mathbf{1}$. By Lemma 8, for almost all $\beta<\kappa, h_{\alpha}(\beta)$ is the value of $\alpha$ by $f(\beta)$. Then, $\left\|\psi\left(h_{\alpha}\right)=\psi\left(f_{\alpha}\right)\right\|=$ 1. We have just proven that $\|(\forall \alpha<\check{\kappa})((\psi(f))(\alpha)=s(\alpha))\|=1$. Since $\psi(f)$ and $s$ are $\check{\kappa}$-sequences, $\|\psi(f)=s\|=\mathbf{1}$.

\section{Boolean algebras in Boolean extensions.}

Let $\mathscr{B}$ be a complete Boolean algebra. Let $\mathscr{D} \in V^{(\mathscr{A})}$ such that $\| \mathscr{D}$ is a Boolean algebra $\|=\mathbf{1}$. We put $\mathscr{D}_{[\mathscr{G}]}=\left\{x \in V^{(\mathscr{D})}:\|x \in \mathscr{D}\|=\mathbf{1}\right\}$. We can make $\mathscr{D}_{[\mathscr{H}]}$ into a Boolean algebra, by defining Boolean operations as follows ;

Let $x, y \in \mathscr{D}_{[\mathscr{s}]}$. Then, there exist uniquely $z_{1}$ and $z_{2}$ such that the followings are $\mathscr{B}$-valid respectively.

1) $z_{1} \in \mathscr{D}$ and $x+{ }_{\mathscr{D}} y=z_{1}$

2) $z_{2} \in \mathscr{D}$ and $-{ }_{\mathscr{D}} x=z_{2}$

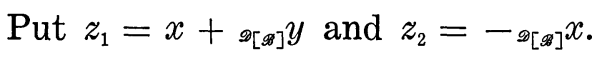

The following lemma is due to Solovay-Tennenbaum [7]

LEMMA 1. $\mathscr{D}_{[\mathscr{B}]}$ is complete iff it is $\mathscr{B}$-valid that $\mathscr{D}$ is complete.

The proof of the following lemma is similar to Lemma 5.2.6 of Solovay-Tennenbaum [7]. So we omit the proof.

LemMa 2. Let $\lambda$ bea regular cardinal. Then the following are equivalent :

1) $\mathscr{B}$ is $\lambda$-saturated, and it is $\mathscr{B}$-valid that $\mathscr{D}$ is $\lambda$-saturated

2) $\mathscr{D}_{[\mathscr{B}]}$ is $\lambda$-saturated.

LEMMA 3. ${ }^{1)}$ If there is a surjection $\Phi$ form $\mathscr{B}$ to $\mathscr{D}_{[\mathscr{B}]}$ such that $\left\|\Phi(b)=\mathbf{1}_{\mathscr{D}}\right\|=b$ and $\left\|\Phi(b)=\mathbf{0}_{\mathscr{D}}\right\|=-b$ for all $b \in \mathscr{B}$, then $\mathscr{D}=\mathbf{2}$ in $V_{(\mathscr{P})}$.

\section{The basic lemma and proof of Theorem 1.}

4.1. Let $\kappa, I$ and $\mathscr{A}$ be as in $\S 2$. Let $\mathscr{B}$ be a complete Boolean algebra. Let $J \in V^{(\mathscr{B})}$ such that $J$ is the ideal on $\check{\kappa}$ generated by $\check{I}$ in $V^{(\mathscr{B})}$. Clearly $\|A \in J\|=\sum_{B \in I}\|A \subseteq B\|$.

LEMMA 1. If $\mathscr{B}$ is $\kappa$-saturated, then it is $\mathscr{B}$-valid that $J$ is nontrivial.

1) cf. Solovay-Tennenbaum [7], p.214. 
Proof. Trivially, $J$ is non-principal. The fact that $J$ is $\kappa$-complete is easily proved by using the following sublemma.

SUBLEMma. If $\mathscr{B}$ is $\kappa$-saurated, then $\|A \in J\|=\|A \subseteq B\|$ for some $B \in I$.

4.2. Let $\mathscr{D} \in V^{(\mathscr{D})}$ such that $\|\mathscr{D}=P(\breve{\kappa}) / J\|^{(\mathscr{P})}=\mathbf{1}$.

BASIC LEMMA. If $\mathscr{B}$ is $\kappa$-saturated, then $\mathscr{D}_{[\otimes]}$ is isomorphic to $\mathscr{B}_{[\&]}^{*}$

Proof. Let $x \in \mathscr{D}_{[\mathscr{B}]}$. Then, there exists $A \in V^{(\mathscr{B})}$ such that $\|x=[A]\|^{(\mathscr{a})}$ $=1$ and $\|A \subseteq \check{\kappa}\|^{(\mathscr{B})}=1$. We define $f_{A} ; \kappa \rightarrow \mathscr{B}$ by $f_{A}(\alpha)=\|\check{\alpha} \in A\|^{(\mathscr{A})}$. Then, $\left\|\psi\left(f_{A}\right) \in \mathscr{B}^{*}\right\|^{(\mathscr{x})}=1$. Put $\Phi(x)=\psi\left(f_{A}\right)$. We must show that the definition of $\Phi(x)$ does not depent on the choice of $A$. So let, $A, B \in P^{(*)}(\kappa)$ such that $\|[A]=[B]\|^{(\mathscr{G})}=1$. Then, $\|A \Delta B \in J\|^{(\mathscr{*})}=1$. $\quad(A \Delta B$ denotes the symmetric difference of $A$ and $B$.) By the sublemma of Lemma 1 , for some $N \in I,\|A \Delta B \subseteq \check{N}\|^{(\mathfrak{m})}=\mathbf{1}$. It follows that if $\alpha \notin N$, then $\|\check{\alpha} \in A\|^{(\mathfrak{g})}$ $=\|\check{\alpha} \in B\|^{(\mathscr{w})}$. Since $N \in I$, for almost all $\alpha<\kappa, f_{A}(\alpha)=f_{B}(\alpha)$. By Lemma 8 of $\S 2$, we have $\left\|\psi\left(f_{A}\right)=\psi\left(f_{B}\right)\right\|^{(\mathscr{A})}=1$. Since $V^{(\mathscr{B})}$ is sepatate $\psi\left(f_{A}\right)=$ $\psi\left(f_{B}\right)$.

$\Phi$ is surjective: Let $y \in \mathscr{B}_{[\propto]}^{*}$. By Lemma 8 of $\S 2$, for some $f \in V^{\boldsymbol{k}}$, $\psi(f)=y$. We may suppose that $f ; \kappa \rightarrow \mathscr{B}$. We define $A \in V^{(\mathscr{P})}$ by $\|\check{\alpha} \in A\|^{(\mathscr{A})}$ $=f(\alpha)$ for $\alpha<\kappa$. Clearly, $\|A \subseteq \check{\kappa}\|^{(\mathscr{a})}=1$. Let $\|x=[A]\|^{(\mathscr{*})}=\mathbf{1}$. Then, $x \in \mathscr{D}_{[\mathscr{R}]}$. By the definition of $\Phi, \Phi(x)=y$.

$\Phi$ is injective: Let $x, y \in \mathscr{D}_{[\mathscr{m}]}$ such that $\Phi(x)=\Phi(y)$. Let $A, B \in V^{(\mathscr{x})}$ be such that $\|x=[A]\|^{(\mathscr{P})}=\|y=[B]\|^{(\mathscr{a})}=\mathbf{1}$. Then, $\psi\left(f_{A}\right)=\Phi(x)=\Phi(y)$ $=\psi\left(f_{B}\right)$. Thus, $f_{A}(\alpha)=f_{B}(\alpha)$ for almost all $\alpha<\kappa$, that is, $\{\alpha<\kappa ;\|\check{\alpha} \in A\|$ $=\|\check{\alpha} \in B\|\} \in I$. By the definition of $J$, we have $\|A \Delta B \in J\|^{(\mathscr{*})}=1$. It follows that $\|x=y\|^{(\mathscr{m})}=\mathbf{1}$.

$\Phi$ is an isomorphism: Let $x, y \in \mathscr{D}_{[\mathscr{w}]}$ be such that $x \leqq y$. Let $A$, $B \in P^{(x)}(\kappa)$ such that $\|x=[A]\|^{(\mathscr{x})}=\|x=[B]\|^{(\mathscr{x})}=1$. Since $x \leqq y$, we have $\|A-B \in J\|^{(\mathscr{a})}=1$. By the sublemma of Lemma 1, for some $N \in I$, $\|A-B \subseteq \check{N}\|^{(\mathscr{m})}=\mathbf{1}$. Thus, if $\alpha \notin N$, then $\|\check{\alpha} \in A\|^{(\mathfrak{m})} \leqq\|\check{\alpha} \in B\|^{(\mathfrak{m})}$. That is, for almost all $\alpha<\kappa, f_{A}(\alpha) \leqq f_{B}(\alpha)$. It follows that $\psi\left(f_{A}\right) \leqq \psi\left(f_{B}\right)$. So, $\Phi(x) \leqq \Phi(y)$.

4.3. Now, we prove Theorem 1. Let $\lambda$ be a regular cardinal less than $\kappa$, and $\gamma$ be a regular cardinal $\lambda \leqq \gamma \leqq \kappa^{+}$. Suppose that $I$ is $\gamma$-saturated and $\mathscr{B}$ is $\lambda$-saturated. Since $\mathscr{B}$ is $\lambda$-saturated and $\lambda<\kappa$, we have $\| N \vDash \mathscr{B}^{*}$ is $\gamma$-saturated $\|^{(\infty)}=\mathbf{1}$. Since $\mathscr{A}$ is $\gamma$-saturated, $\| \check{\gamma}$ is a cardinal $\|^{(\infty)}=\mathbf{1}$. 
By Lemma 9 of $\S 2$ and the fact that $\lambda \leqq \gamma$, we have $\| \mathscr{B}^{*}$ is $\gamma$-saturated $\|^{(\mathscr{A})}$ = 1. By Lemma 2 of $\S 3$, we have $\mathscr{B}_{[\&]}^{*}$ is $\check{\gamma}$-saturated. By the basic lemma, $\mathscr{D}_{[\mathscr{B}]}$ is $\gamma$-saturated.

Again, by Lemma 2 of $\S 3, \| \mathscr{D}$ is $\check{\gamma}$-saturated $\|^{(\mathscr{m})}=\mathbf{1}$. That is, $\| J$ is $\check{\gamma}$-saturated $\|^{(\mathscr{g})}=\mathbf{1}$.

Remark. In the case when $\kappa$ is measurable and $I$ is a non-trivial prime ideal on $\kappa, \mathscr{A}=P(\kappa) / I=2$. So we may consider $N$ as a transitive class in the real world.

The following theorem can be proved by using the basic lemma.

THEOREM (Lévy-Solovay [3]). Let $\kappa$ be a measurable cardinal and I be a non-trivial prime ideal on $\kappa$. Let $\mathscr{B}$ be a complete Boolean algebra such that $\operatorname{card}(\mathscr{B})<\kappa$. Then, it is $\mathscr{B}$-valid that $J$ is a non-trivial prime ideal on $\kappa$.

Proof. By the basic lemma, $\mathscr{D}_{[\mathscr{B}]}$ is isomorphic to $\mathscr{B}^{*}$. Let $\Phi$ be an isomorphism from $\mathscr{D}_{[\mathscr{B}]}$ to $\mathscr{B}^{*}$. Define $\Psi ; \mathscr{B} \rightarrow \mathscr{B}^{*}$ by $\psi(b)=b^{*}$. Trivially $\Psi$ is injective. Let $\psi(f) \in \mathscr{B} *$. We may suppose that $f ; \kappa \rightarrow \mathscr{B}$. Since $\operatorname{card}(\mathscr{B})<\kappa$, there is the unique $b \in \mathscr{B}$ such that $f(\alpha)=b$ for almost all $\alpha<\kappa$. Thus, $\psi(f)=\Psi(b)$. It follows that $\Psi$ is bijective. Let $i=\Phi^{-1} \circ \Psi$. Let $b \in \mathscr{B}$. By easy computations, we have $\left\|\left(\Phi^{-1} \circ \Psi\right)(b)=\mathbf{1}_{\mathscr{D}}\right\|=b$ and $\left\|\left(\Phi^{-1} \circ \Psi\right)(b)=\mathbf{0}_{\mathscr{D}}\right\|=-b$. By Lemma 3 of $\S 3$, we have $\|\mathscr{D}=\mathbf{2}\|=\mathbf{1}$. That is, $\| J$ is prime $\|^{(\mathscr{P})}=\mathbf{1}$.

\section{Proofs of Theorem 2 and 3 .}

5.1. Let $\mathscr{B}$ a complete Boolean algebra, and $\pi$ be an automorphism of $\mathscr{B}$. Then, $\pi$ induces the automorphism $\pi_{*}$ of $V^{(\mathscr{B})}$.

LEMMA 1. Let $\phi\left(v_{0}, \cdots, v_{n-1}\right)$ be a set-theoretical formula, and let $x_{0}, \cdots, x_{n-1}$ be elements of $V^{(\mathscr{B})}$. Then,

$$
\| \phi\left(\pi_{*}\left(x_{0}\right), \cdots, \pi_{*}\left(x_{n-1}\right) \|=\pi\left(\left\|\phi\left(x_{0}, \cdots, x_{n-1}\right)\right\|\right) .\right.
$$

Proof. The lemma is easily proved by induction on the length of $\phi$. An element $x$ of $V^{(\mathscr{x})}$ is called $\pi$-invariant if $x=\pi_{*}(x) . \quad x$ is called invariant if $x$ is $\pi$-invariant for all automorphisms $\pi$ of $\mathscr{B}$. For example, $\check{x}$ is invariant for each $x \in V$.

By using Lemma 1, the following lemma is trivial. 
LEMMA 2. Let $\phi\left(v_{0}, \cdots, v_{n-1}\right)$ be a set-theoretical formula, and let $x_{0}, \cdots, x_{n-1}$ be invariant elements of $V^{(*)}$. Then, $\left\|\phi\left(x_{0}, \cdots, x_{n-1}\right)\right\|=$ $\pi\left(\left\|\phi\left(x_{0}, \cdots, x_{n-1}\right)\right\|\right)$.

5.2. Let $\mathscr{B}$ be a Boolean algebra. We consider the following condition $(*)$.

(*) $\mathbf{0}$ and $\mathbf{1}$ are the only invariant elements of $\mathscr{B}$.

We say that a Boolean algebra $\mathscr{B}$ is homogeneous if: for every $\mathbf{0}<b$, $c<1$, there exists an automorphism $\pi$ such that $\pi(b)=c$. Clearly, if $\mathscr{B}$ is homogeneous, then $\mathscr{B}$ satisfies the condition (*).

LEMMA 3. Let $\phi\left(v_{0}, \cdots, v_{n-1}\right)$ be a set-theoretical formula, and $\mathscr{B}$ be a complete Boolean algebra satisfying the condition (*). Let $x_{0}, \cdots, x_{n-1}$ be invariant elements of $V^{(\mathfrak{*})}$. Then, $\left\|\phi\left(x_{0}, \cdots, x_{n-1}\right)\right\|=\mathbf{0}$ or $\mathbf{1}$.

Proof. Suppose not. Put $\left\|\phi\left(x_{0}, \cdots, x_{n-1}\right)\right\|=b$. Then, $\mathbf{0}<b<\mathbf{1}$. Since $\mathscr{B}$ satisfies the condition $\left(^{*}\right)$, there exists an automorphism $\pi$ such that $\pi(b) \neq b$. Then,

$$
\pi\left(\left\|\phi\left(x_{0}, \cdots, x_{n-1}\right)\right\|\right) \neq\left\|\phi\left(x_{0}, \cdots, x_{n-1}\right)\right\| \text {. }
$$

This contradicts to Lemma 2.

Let $\mathscr{P}$ be a partially ordered set. We make $\mathscr{P}$ into a topological space by taking sets of the form

$$
U_{p}=\{q \in \mathscr{P} ; q \leq p\}
$$

as a basis for the open sets. Let $\mathscr{B}_{\mathscr{P}}$ be the complete Boolean algebra of regular open sets of $\mathscr{P}$. Let $\pi$ be an automorphism of $\mathscr{P}$. Then, $\pi$ induces the automorphism $\bar{\pi}$ of $\mathscr{B}_{\mathscr{g}}$ by $\bar{\pi}(U)=\{\pi(p) ; p \in U\}$.

LEMMA 4. Let $\mathscr{P}$ be a partially ordered set satisfying the condition $(* *)$.

(**) Let $p$ and $q$ be elements of $\mathscr{P}$. Then, there is an automorphism $\pi$ of $\mathscr{P}$ such that $\pi(p)$ and $q$ are compatible.

Then, $\mathscr{B}_{g}$ satisfies the condition (*).

Proof. Suppose not. Then, there exists an element $\mathbf{0}<U<\mathbf{1}$ of such that $\pi(U)=U$ for all automorphisms $\pi$ of $\mathscr{B}_{g}$. Let $p$ and $q$ be elements of $\mathscr{P}$ such that $p \in U$ and $q \in$ interior $(-U)$. Since $\mathscr{P}$ satisfies the condition $(* *)$ there exists an automorphism $\pi$ of $\mathscr{P}$ such that $\pi(p)$ and $q$ are compatible. Then, there exists an element $r$ of $\mathscr{P}$ such that 
$r \leq \pi(p)$ and $r \leq q$. Since $\bar{\pi}(U)=U, \pi(p) \in U$. By the fact that $U$ is open, $r \in U$. Since $q \in$ interior $(-U), r \in-U$. This is a contradiction.

5.3. Let $\kappa$ be an uncountable cardinal, and let $I$ be a non-trivial ideal on $\kappa$. Let $J \in V^{(\mathscr{x})}$ be the ideal generated by $\check{I}$ inside $V^{(\mathfrak{x})}$.

LEMMA $5 . \quad J$ is invariant.

Proof. Let $\pi$ be an automorphism of $\mathscr{B}$. By Lemma $1, \| \pi_{*}(J)$ is the ideal on $\pi_{*}(\check{\kappa})$ generated by $\pi_{*}(\check{I}) \|=1$. Since $\check{\kappa}$ and $\check{I}$ are invariant, $\| \pi_{*}(J)$ is the ideal on $\check{\kappa}$ generated by $\check{I} \|=\mathbf{1}$. Hence, $\left\|\pi_{*}(J)=J\right\|=\mathbf{1}$. Since $V^{(\mathscr{B})}$ is separate, $\pi_{*}(J)=J$.

5.4. Let $\kappa$ and $I$ be as in 5.3. Suppose that $I$ is $\kappa$-saturated.

LEMMA 6. Let $\mathscr{B}$ be a complete Boolean algebra satisfying the condition (*). Suppose that $\operatorname{sat}(\mathscr{B})=\kappa$. Then, it is $\mathscr{B}$-valid that $J$ is not $\kappa$-saturated.

Proof. Suppose not. Since $\mathscr{B}$ satisfies the condition $(*), \| J$ is $\check{\kappa}$ saturated $\|^{(\mathscr{A})}=1$ by Lemma 3 and Lemma 5. Let $\mathscr{D} \in V^{(\mathscr{P})}$ such that $\|\mathscr{D}=P(\kappa) / J\|^{(\mathscr{O})}=1$. By Lemma 2 of $\S 3, \mathscr{D}_{[\mathscr{G}]}$ is $\kappa$-saturated. By the basic lemma, $\mathscr{B}_{[\propto]}^{*}$ is $\kappa$-saturated. Then, $\| \mathscr{B}^{*}$ is $\kappa$-saturated $\|^{(\mathscr{\alpha})}=\mathbf{1}$. Clearly, $\| N \vDash \mathscr{B}^{*}$ is $\kappa$-saturated $\|^{(\infty)}=\mathbf{1}$. Choose $f \in V^{\kappa}$ so that $\psi(f)=\check{\kappa}$. We may suppose that $f: \kappa \rightarrow \kappa$. The, for almost all $\alpha<\kappa$, $\mathscr{B}$ is $f(\alpha)$ saturated. Thus, sat $(\mathscr{B})<\kappa$. This contradicts to the assumption of $\mathscr{B}$.

Now Theorem 2 is a corollary of Lemma 6 .

5.5. Let $\kappa$ be a measurable cardinal, and $I$ be a non-trivial prime ideal on $\kappa$.

LEMMA 7. $2^{\kappa}<\kappa^{*}$.

Proof. Since $P(\kappa)=P(\kappa) \cap N, 2^{\kappa} \leq 2^{\kappa(N)}$. On the other hand $\kappa^{*}$ is measurable in $N$, so $\kappa^{*}$ is strongly inaccessible in $N$. Hence, $2^{\kappa(N)}<\kappa^{*}$. Thus, $2^{\kappa}<\kappa^{*}$.

Theorem 3 is a corollary of the following lemma.

LEMma 8. Let $\mathscr{B}$ be a complete Boolean algebra satisfying the condition (*). Assume that $\operatorname{sat}(\mathscr{B})=\kappa$. Let $J \in V^{(\mathscr{B})}$ be the ideal on $\check{\kappa}$ generated by $\check{I}$ inside $V^{(\mathscr{B})}$. Then, it is $\mathscr{B}$-valid that $J$ is not $\kappa^{+}$-saturated.

Proof. By using Lemma 7, the proof can be carried out analogously 
to the proof of Lemma 6. (Note that $\kappa^{+}<\kappa^{*}$ by Lemma 7.).

5.6. We give an application of Lemma 8. Let $\kappa$ and $I$ be as in 5.5. We consider the following partially ordered set $\mathscr{P} ; p \in \mathscr{P}$ if

1) $p$ is a function

2) the domain of $p$ is a finite subset of $\kappa \times \omega$

3) the range of $p \subseteq \kappa$

4) $p(\langle\alpha, n\rangle)<\alpha$ whenever $\langle\alpha, n\rangle \in \operatorname{domain}(p)$.

The ordering of $\mathscr{P}$ is $\subseteq$. Clearly, $\mathscr{P}$ satisfies the condition $(* *)$.

LEMMA 9. ${ }^{1)} \quad \operatorname{Sat}\left(\mathscr{B}_{\mathscr{g}}\right)=\kappa . \quad\left\|\kappa=\boldsymbol{\aleph}_{1}^{\left(\mathscr{P}_{\mathscr{g}}\right)}\right\|=\mathbf{1}$.

By the theorem of $\S 2$ and Lemma 9, $\| \breve{\kappa}$ has no $\breve{\kappa}$-saturated non-trivial ideal on $\kappa \|=1$. On the other hand, by Lemma 8 we have $\| J$ is not an

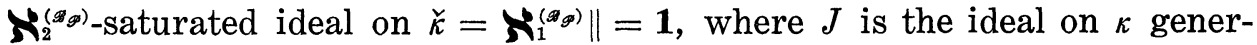
ated by $\check{I}$ inside $V^{(\mathscr{G g})}$.

\section{REFERENCES}

[1] K. Kunen, Some applications of iterated ultrapowers in set theory. Annals of Math. Logic 1. (1970) 179-227.

[2] K. Kunen and J. B. Paris, Boolean extensions and measurable cardinals. Annals Math. Logic Vol. 2 No. 4 (1971) 359-377.

[ 3 ] A. Lévy and R. Solovay, Measurable cardinal and the continuum hypothesis, Israel Journal of Math. Vol. 5 (1967) 234-248.

[4] D. Scott and R. Solovay, Boolean-valued models for set theory, Proc. 1967. U.C.L.A. Summer Institute, to appear.

[ 5] R. Solovay, Real-valued measurable cardinals. Proc. of symposia in pure math. Vol. XIII, Part I (1971) 397-428.

[ 6 ] R. Solovay, A model of set-theory in which every set of reals is Lebesgue measurable. Annals of Math. 92 (1970) 1-56.

[ 7 ] R. Solovay and S. Tennenbaum, Iterated Chen extensions and Souslin's problem. Annals of Math. Vol. 94 No. 2 201-245.

[ 8 ] K. L. Prikry, Changing measurable into accessible cardinals. Dissertationes Math. (Rzprawy Mat.) 68 (1970).

Nagoya University

1) See Solovay [6], p.15. 